\title{
The method and devices of autocompensation of phase distortions of direct digital synthesizers of signal formers of georadars
}

\author{
$N V$ Dorofeev $^{1, *}, A V$ Grecheneva $^{1}, O R$ Kuzichkin $^{1}, D I$ Surzhik $^{2}$, and $R V$ Romanov $^{2}$ \\ ${ }^{1}$ Belgorod State University, 85 Pobedy St, Belgorod, 308015, Russia \\ ${ }^{2}$ Vladimir State University, 87 Gor'kogo St, Vladimir, 600000, Russia
}

\begin{abstract}
The method for isolating the clock frequency of direct digital synthesizers, as well as algorithms and devices for isolating and automatically compensating phase distortions, that significantly improve the spectral characteristics of the formers of the probing signals of georadars used for geodynamic monitoring are considered in the article. The method is substantiated, explanatory oscillograms, spectrograms, and the results of the experimental researches, confirming the authenticity, are presented.
\end{abstract}

\section{Introduction}

Georadar probing is one of the effective methods of non-destructive testing in the implementation of geodynamic monitoring [1-3]. It is applied at the stages of preliminary analysis of the researched media during engineering geological, ecological and permafrostglaciological researches, the depth of which does not exceed several tens of meters. Its use ensures the continuity of measurements and clearly establishes the position of the boundaries of the sections of the soil. It also allows to reduce economic and time costs by an order in comparison with other control methods that require opening of the researched surface or drilling of wells. Other its major advantages are high performance and depth resolution, as well as mobility and manufacturability. The modern georadar locating installation is a stationary or mobile georadar. Its main structural blocks are the former of probing signals, the receiving-transmitting module and the unit for processing the reflected signals [1].

One of the most important tasks in the practical implementation of a georadar is to ensure the coherence and stability of oscillations generated by the former of probing signals. The authors' researches has shown that the effective option of implementation of this device, which provides these requirements, is the use of direct digital synthesizers (DDS) [4-8] and various ways for constructing the formers of probing signals based on them. In figure 1 a block diagram of the DDS is given, on which the following designations are adopted: CG clock generator, PA - phase accumulator, ROM - read-only memory, DAC - digital-toanalog converter, LPF - low-pass filter, $f_{\text {clk }}$ - clock frequency, $K$ - binary code of frequency, $f_{\text {DDS }}$ - synthesized frequency.

\footnotetext{
* Corresponding author: dorofeevnv@yandex.ru
} 


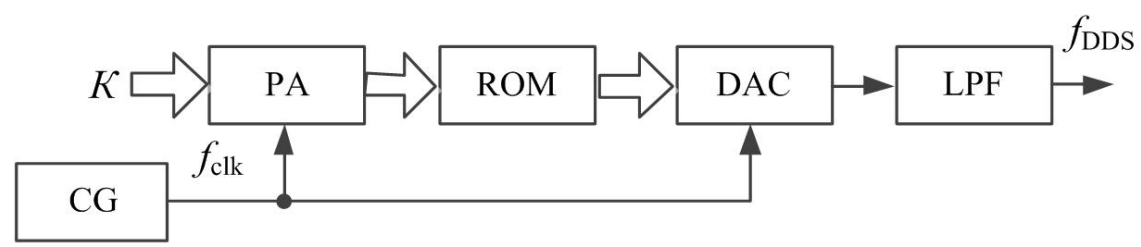

Fig. 1. Block diagram of the DDS.

Modern DDSs have a significant disadvantage, which partially limiting their use and associated with the presence in the spectrum of the synthesized signal of phase noise and parasitic spectral components (PSCs) which the level at minus 60 - minus $80 \mathrm{~dB}$. Known methods for improving the spectral characteristics of the DDSs have limited application and are not effective enough.

\section{Justification of the possibility of applying the method of automatic compensation of phase distortions}

It is proved that PSCs and phase noises, representing the greatest complexity for suppression, lead to undesirable dynamic deviations of the fronts of the output signal of its DAC. This process is accompanied by a change in the width of the "steps" and the displacement relative to the ideal location on the time scale [9]. In the time domain this process corresponds to the presence of phase distortions - the parasitic phase modulation of the useful signal.

The fact of the presence of parasitic phase modulation makes allows us to consider automatic compensation as a method for reducing the phase distortions of the output distortion of the DAC of the DDS [10,11]. It is assumed that the output signal of the DDS is an additive mixture of the useful signal and the result of the amplitude-phase distortions and can be considered as a complex AM-PM oscillation in the form of a sum of AM and PM components. Automatic compensation of phase distortion (ACPD) is accomplished by reducing undesirable deviations of the signal phase in the automatic mode by introducing their antiphase change formed by the device itself from the original signal.

Known devices of autocompensation cannot solve the problem of reducing phase distortions in the output signal of the DDS. This is due to the difference between the input clock signal and output step signal of the DAC of the DDS in terms of amplitude, frequency and shape. This makes it difficult to compare and isolate the phase distortions $[10,11]$.

Consider features of isolation and the subsequent autocompensation of phase distortions of the output signal of DDS. The spectrum of its DAC in the general case contains harmonics of the main frequency $k f_{\mathrm{DDS}}$, combinational components with frequencies $m f_{\text {clk }} \pm$ $k f_{\mathrm{DDS}}$, noise and discrete components, and the envelope at frequencies that are multiples of the clock frequencies $\left(m f_{\text {clk }}\right)$ takes zero values [4-8]. As an example, figure 2 shows the spectrum of the output signal of the DAC of the DDS AD9854 (manufactured by Analog Devices) with a synthesized frequency of $3.1 \mathrm{MHz}$ and a clock frequency of $30 \mathrm{MHz}$. 


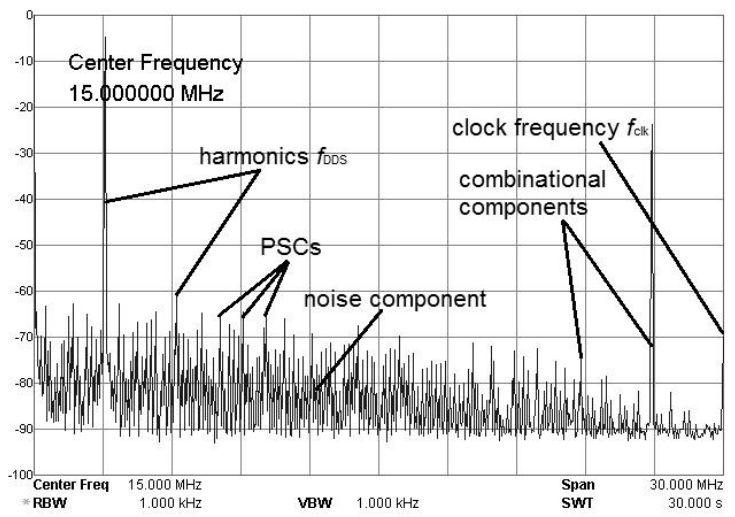

Fig. 2. Spectrum of the output signal of the DAC of DDS AD9854.

In the output spectrum of the DAC of the DDS the clock frequency is absent. However, the presence of information about this frequency in the time domain is evident - the width of the "steps" of the analog signal corresponds to the period of the meander. In this regard, the structural diagram of the DDS can be equivalently represented as a balance modulator (BM) with an adder (Add) - figure 3. In the output spectrum of balance modulator, as well as in the output spectrum of the DDS, only side bands are present, and the carrier frequency components are absent.

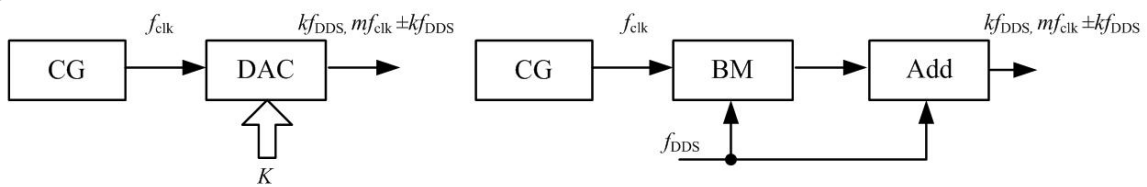

Fig. 3. Structural diagram of the DDS and its equivalent model.

In the presence of phase distortions of the output signal of the DDS, all the components of the spectrum are modulated according to the same law as the synthesized frequency, but with other modulation indices. The clock frequency is constant and has the same law of parasitic phase modulation as the output signal of the DDS. By processing the output signal of the DAC in a certain way and selecting the clock frequency in the output spectrum, it is possible to automatically compensate the phase distortions of the output signal of the DDS at this frequency.

To isolate the clock frequency, first need to separate the "steps" of the DAC output signal. Such a signal transformation can be performed in a differentiating circuit. The shape of the signal from the output of the DAC after passing through this circuit $s_{\mathrm{DC}}(t)$ represents a sequence of several alternating voltage jumps of positive and negative polarity with an envelope varying in a cosine law.

The signal $s_{\mathrm{DC}}(t)$ can be represented as the sum of two components $s_{1}(t)$ and $s_{4}(t)$, and each of them in turn is the sum of two components: $s_{1}(t)=s_{2}(t)+s_{3}(t)$ for the positive range of the function $s_{\mathrm{DC}}(t)$ and $s_{4}(t)=s_{5}(t)-s_{6}(t)$ for the negative region. And the components $s_{3}(t)$ and $s_{6}(t)$ are envelopes, and $s_{2}(t)$ and $s_{5}(t)$ - high-frequency filling.

To isolate the clock frequency from the spectrum of output signal of the differentiating circuit, a nonlinear transformation of its spectrum is necessary. The device that performs such a conversion is a full-wave rectifier, whose output signal is defined as the module of input signal $s_{\mathrm{FWR}}(t)=\left|s_{\mathrm{DC}}(t)\right|$.

In figure 4 schematically shows the spectrums of the output signals of the differentiating circuit and the full-wave rectifier, as well as their components, where the symbol $\alpha$ denotes other frequency components of the spectrums. 

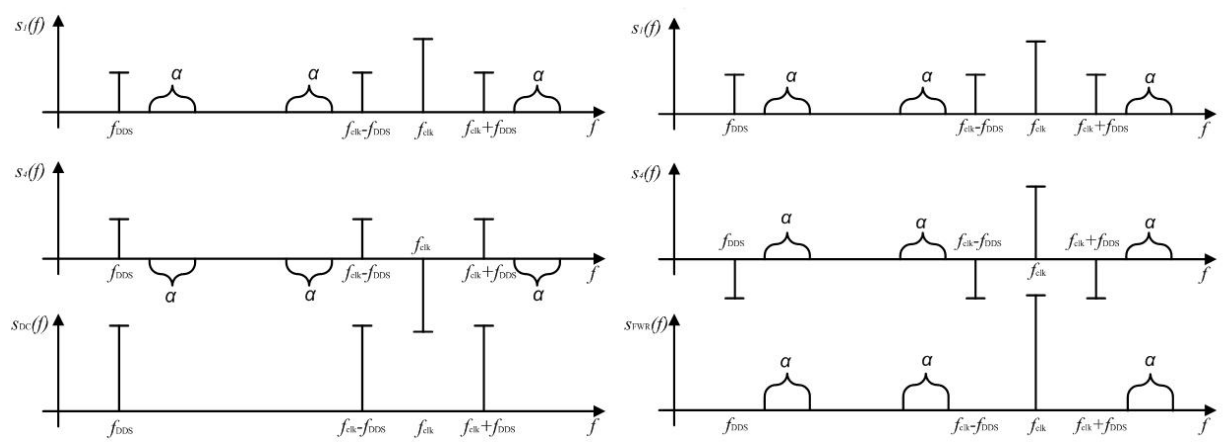

Fig. 4. Spectrums of the output signals of the differentiating circuit and the full-wave rectifier, as well as their components.

In the output spectrum of the differentiating circuit there are antiphase components with a frequency $f_{\text {clk }}$ which mutually compensating each other. The components of the output frequency of the DDS and the combinational components are in phase and therefore are summed. After passing the full-wave rectifier, the signal $s_{1}(t)$ and its spectrum do not change. The signal $s_{4}(t)$ turns out to be inverted, and accordingly the spectrum of the this signal becomes inverted. As a result, the output spectrum of the nonlinear device contains in-phase signal components $s_{1}(t)$ and $s_{4}(t)$ with frequencies $f_{\text {clk }}$ that are added, and the spectral components with frequencies $f_{\text {DDS }}$ and the combination components cancel each other out.

On the basis of the proposed method, algorithms and structural diagrams of devices for isolating phase distortions of the DDS and forming the compensating signal of the ACPD are developed [10,11]. One of the variants of the algorithm and the device is shown in figure 5. The following designations are accepted on the diagram: LPF DDS - output lowpass filter of the DDS, Tr - T-trigger, DC - differentiating circuit, A - amplifier, FWR - fullwave rectifier, $\mathrm{PD}$ - phase detector, $\mathrm{CD}$ - control device, RT - reference tract, IT information tract, CT - control tract.
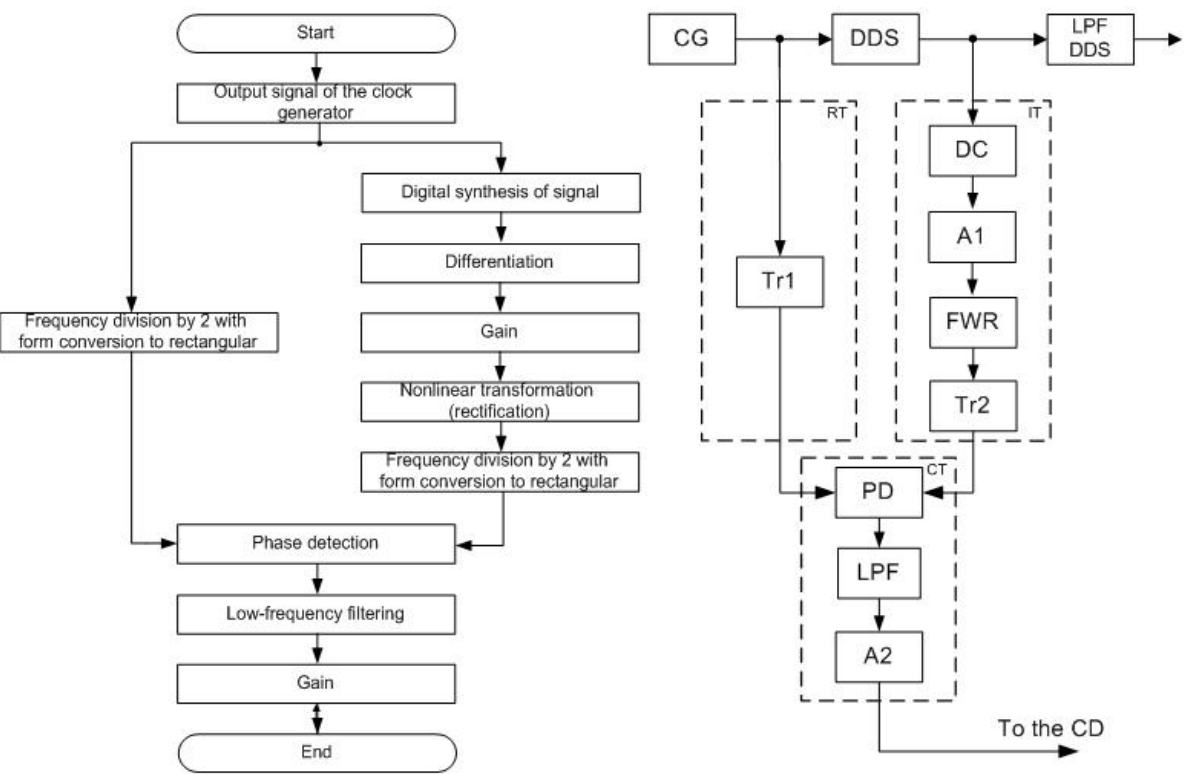

Fig. 5. Algorithm and block diagram of the device for isolating phase distortions of the DDS and forming the compensating signal of the ACPD. 


\section{Experimental research of the method of isolation of the clock frequency of the DDS}

Practical confirmation of the authenticity of the proposed method for isolating the clock frequency of the DDS, algorithms and devices is realized (figure 5). An experimental investigation of output signals and spectrums of the blocks of autocompensator of phase distortions, which specially developed for the DDS AD9854, has been carried out. This synthesizer contains a 48-bit phase accumulator, a 14-bit ROM, a 12-bit DAC, and a builtin oscillator with a clock frequency of $30 \mathrm{MHz}$.

The investigated method of isolation of the clock frequency of the DDS is realized by the information tract of the autocompensator. As its differentiating circuit, a high-pass filter of the first order is used. The amplifier is represented by two cascades on bipolar transistors KT368A in the circuit of inclusion with a common emitter. The full-wave rectifier is realized by the circuit on the low-noise operational amplifier AD8055. Trigger of information tract is based on the D-flip-flop 74VHC74.

In figure 6 shows the spectrum of the output signal of the DAC of DDS AD9854 with a frequency of $7.2 \mathrm{MHz}$ and the result of its passage through the differentiating circuit and full-wave rectifier. This figure clearly illustrates the proposed method for isolating the clock frequency of the DDS for the subsequent autocompensation of its phase distortions.

In the output spectrum of the DAC of DDS AD9854, in addition to the main frequency of $7.2 \mathrm{MHz}$, there are harmonics and a lot of discrete PSCs at the level minus 41- minus 60 $\mathrm{dB}$. In addition, the nonideality of the digital-to-analog conversion is accompanied by the fact that in the output spectrum of the DAC there is a spectral component of the clock frequency of the level minus $42 \mathrm{~dB}$. After passing the output signal of the DAC of DDS AD9854 through the differentiating circuit, a redistribution of the amplitudes of the harmonics of the main frequency and discrete PSCs is observed in its spectrum. As a result of the nonlinear conversion of this signal, a first harmonic of the main frequency of the DDS 7.2 MHz is weakens significant (from minus $25 \mathrm{~dB}$ to minus $51 \mathrm{~dB}$ ). In this case, the levels of the even harmonic and the combinational component reach minus $36 \mathrm{~dB}$ and minus $44 \mathrm{~dB}$, respectively, and a significant increase in the spectral component of the clock frequency of $30 \mathrm{MHz}$ occurs.

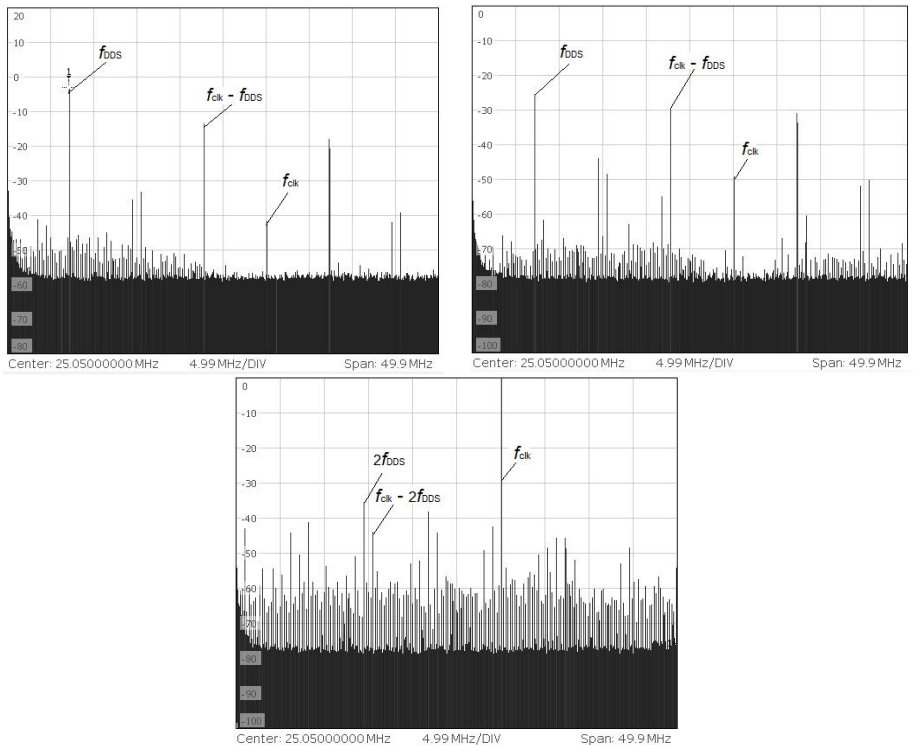

Fig. 6. Signal spectrums at the outputs of the DAC of the DDS AD9854, the differential circuit DC2 and the full-wave rectifier FWR 


\section{Conclusion}

The carried out experimental researches, as well as the results of mathematical and circuit simulations of direct digital synthesizers and formers of probing signals of georadars on their basis testify to the authenticity of the proposed method, algorithms and devices for isolating and automatic compensation of phase distortions of the output signal of the DDS. Based on the results of the analysis, it was found that the maximum practicable reduction in the PSCs level is $15 \mathrm{~dB}$, and the level of phase noise is about $10-13 \mathrm{~dB}$. It is due to the presence of the noise contribution of the autocompensator.

\section{Acknowledgments}

The work was carried out as part of the research on the project, support by the Ministry of Education and Science of the Russian Federation No. 5.3606.2017/PCH.

\section{References}

1. Khmelevskoy V K 1989 Handbook of Exploration Geophysics vol 2, ed V K Khmelevskoy and V M Bondarenko (Moscow: Nedra) p 438

2. Fedynsky V V 1964 Exploration geophysics (Moscow: Nedra) p 677

3. Kuzichkin O, Mikhaleva E, Dorofeev N and Romanov R 2017 IEEE 9th Int. Conf. on IDAACS 21-23 Sept. vol 1 (Bucharest: IEEE) pp 227-230

4. Vankka J and Halonen K 2000 Direct Digital Synthesizers: Theory, Design and Applications (Helsinki: University of Technology) p 208

5. Kroupa V F 2003 Phase Lock Loops and Frequency Synthesis (John Wiley \& Sons, Ltd) p 320

6. Goldberg B 1999 Digital Frequency Synthesis Demystified DDS and Fractional-N PLLs (Eagle Rock VA: LLH Technology Publishing) p 355

7. Ridiko L I 2001 Components and technologies vol 17 (St. Petersburg: Publisher Fainstreet) pp50-56

8. Kester W 2007 Analog-to-digital conversion (Moscow: Technosphere) p 1016

9. Peng L 2009 Jitter Electronic components vol 10 pp 81-85

10. Surzhik D I, Kurilov I A, Kuzichkin O R, Vasilyev G S and Kharchuk S M 2015 Int. Conf. On Control and Communications (Omsk: IEEE) pp 1-7

11. Vasilyev G S, Kuzichkin O R, Kurilov I A and Surzhik D I 2016 International Int. Conf. On Control and Communications (Moscow: IEEE) pp 1-6 